\title{
Sex difference in cerebrospinal fluid/ blood albumin quotients in patients with schizophreniform and affective psychosis
}

\author{
Sophie Meixensberger ${ }^{1,2+}$, Karl Bechter ${ }^{3 \dagger}$, Rick Dersch ${ }^{4}$, Bernd Feige ${ }^{1,2}$, Simon Maier ${ }^{1,2}$, Miriam A. Schiele ${ }^{2}$, \\ Kimon Runge ${ }^{1,2}$, Dominik Denzel ${ }^{2}$, Kathrin Nickel ${ }^{1,2}$, Derek Spieler ${ }^{5}$, Horst Urbach $^{6}$, Harald Prüss ${ }^{7,8}$, \\ Katharina Domschke ${ }^{2,9}$, Ludger Tebartz van Elst ${ }^{1,2^{*}}$ and Dominique Endres ${ }^{1,2^{*}}$ (1)
}

\begin{abstract}
Background: The importance of cerebrospinal fluid (CSF) diagnostics for psychiatry is growing. The CSF/blood albumin quotient $\left(\mathrm{Q}_{\mathrm{Alb}}\right)$ is considered to be a measure of the blood-CSF barrier function. Recently, systematically higher $\mathrm{Q}_{\mathrm{Alb}}$ in males than in females was described in neurological patients. The aim of this study was to investigate whether a sex difference could also be detected in a well-characterized psychiatric cohort.

Methods: The patient cohort comprised 989 patients, including 545 females and 444 males with schizophreniform and affective syndromes who underwent CSF diagnostics, including $Q_{\text {Alb }}$ measurement. The basic CSF findings and antineuronal autoantibody data of this cohort have already been published. This re-analysis employed analysis of covariance with age correction for $\mathrm{Q}_{\mathrm{Alb}}$ mean values and chi'-testing for the number of increased age-corrected $\mathrm{Q}_{\mathrm{Alb}}$ levels to investigate sex differences in $\mathrm{Q}_{\text {Alb }}$.

Results: The $\mathrm{Q}_{\text {Alb }}$ levels were elevated above reference levels by $18 \%$ across all patients, and a comparison between male and female patients revealed a statistically significant sex difference, with increased values in $26 \%$ of male patients and a corresponding rate of only $10 \%$ in female patients $\left(c h i^{2}=42.625, p<0.001\right)$. The mean $\mathrm{Q}_{\mathrm{Alb}}$ values were also significantly higher in males $\left(6.52 \pm 3.69 \times 10^{-3}\right)$ than in females $\left(5.23 \pm 2.56 \times 10^{-3} ; F=52.837, p<0.001\right)$.

Discussion: The main finding of this study was a significantly higher $\mathrm{Q}_{\text {Alb }}$ level in male compared to female patients with psychiatric disorders, complementing previously described sex differences in neurological patient cohorts. This result indicates bias from some general factors associated with sex and could be partly explained by sex differences in body height, which is associated with spine length and thus a longer distance for CSF flow within the subarachnoid space down the spine from the occipital area to the lumbar puncture site in males compared to females. Hormonal influences caused by different estrogen levels and other sex-specific factors could also play a relevant role. The significance of the study is limited by its retrospective design, absence of a healthy control group, and unavailability of exact measures of spine length.
\end{abstract}

Keywords: Cerebrospinal fluid, Albumin quotient, Protein, Psychosis, Sex

*Correspondence: tebartzvanelst@uniklinik-freiburg.de; dominique. endres@uniklinik-freiburg.de

†'Sophie Meixensberger, Karl Bechter shared first

${ }^{1}$ Section for Experimental Neuropsychiatry, Department of Psychiatry and Psychotherapy, Medical Center-University of Freiburg, Faculty of Medicine, University of Freiburg, Freiburg, Germany

Full list of author information is available at the end of the article

\section{Background}

Interest in the immunological pathways underlying the etiology and pathophysiology of a subgroup of patients with mental disorders has increased in recent years. The cerebrospinal fluid (CSF) is a dynamic,

c) The Author(s) 2020. This article is licensed under a Creative Commons Attribution 4.0 International License, which permits use, sharing, adaptation, distribution and reproduction in any medium or format, as long as you give appropriate credit to the original author(s) and the source, provide a link to the Creative Commons licence, and indicate if changes were made. The images or other third party material in this article are included in the article's Creative Commons licence, unless indicated otherwise in a credit line to the material. If material is not included in the article's Creative Commons licence and your intended use is not permitted by statutory regulation or exceeds the permitted use, you will need to obtain permission directly from the copyright holder. To view a copy of this licence, visit http://creativeco mmons.org/licenses/by/4.0/. The Creative Commons Public Domain Dedication waiver (http://creativecommons.org/publicdomain/ zero/1.0/) applies to the data made available in this article, unless otherwise stated in a credit line to the data. 
metabolically active secretion that can provide important information pertaining to inflammatory changes involving the brain [1]. Therefore, diagnostic examinations of CSF composition have been recommended increasingly in the study of severe mental disorders [2]. The blood-brain barrier (BBB) is the primary interface dividing peripheral circulation from the central nervous system (CNS) [3]. Its most notable component is the cerebral microvascular endothelium, which functions as a diffusion barrier with selective uptake and efflux transporters and specific vesicular transport [36]. Endothelial tight junctions restrict the paracellular pathway between the blood and brain for polar solutes $[5,7]$. The CSF/blood albumin quotient $\left(\mathrm{Q}_{\mathrm{Alb}}\right)$ has been considered the gold standard for assessment of the BBB function [7]. However, a complicated system of three or more brain barriers and interfaces is now recognized [8-11]. Therefore, $\mathrm{Q}_{\mathrm{Alb}}$ levels are now considered a measure of the overall blood-CSF-barrier (BCSFB) $[12,13]$ in the framework of the flow-diffusion model of BCSFB (dys-)function originally proposed by Reiber [13-15]. Thus, the overall BCSFB is a construct created to explain the complicated interrelationship between CSF and surrounding tissues and the many dynamic influences of exchange involved, as most recently seen in analysis of CSF composition for clinical purposes. Albumin is synthesized predominantly in the liver, from which it rapidly enters the bloodstream and enters CSF circulation mainly during the process of CSF production by the choroid plexus in the brain ventricles, with albumin representing approximately $80 \%$ of normal CSF total protein [12]. However, recent research results also demonstrated that under certain circumstances albumin can also be produced in the brain by microglial cells $[16,17]$. With increasing age, the permeability of the $\mathrm{BBB} / \mathrm{BCSFB}$ increases, whereas CSF production and thus the CSF turnover rate declines [12, 13]. Both of these factors contribute to a distinct age-dependent increase of $\mathrm{Q}_{\mathrm{Alb}}$; thus, $\mathrm{Q}_{\mathrm{Alb}}$ reference values are generally corrected for age $[12,13]$. Moreover, $Q_{A l b}$ could be influenced by other factors such as body weight, body mass index, a reduction in CSF production from reasons other than age, alcohol and nicotine consumption $[1,14]$. Interestingly, an influence of sex on $Q_{\text {Alb }}$ was recently also noticed and discussed in two papers that investigated patient cohorts with a spectrum of neurological disorders $[18,19]$.

The aim of this study was to investigate whether similar sex differences in $\mathrm{Q}_{\mathrm{Alb}}$ can be detected in a cohort of psychiatric patients by reanalyzing the data of a recently published study of a well-characterized sample of patients with schizophreniform and affective psychosis [20].

\section{Methods}

The retrospective data analyses received approval from the local ethics committee of the University of Freiburg (EK Fr 396/18). Between January 2006 and November 2019, 992 patients were investigated [20], of which only those patients who had been tested for $\mathrm{Q}_{\mathrm{Alb}}$ were analyzed in the current study $(\mathrm{N}=989)$.

\section{Female and male patient groups}

All patients received a lumbar puncture (LP) for the organic clarification of their mental disorder. Schizophreniform syndromes (according to the International Statistical Classification of Diseases and Related Health Problems criteria, version 10 [ICD-10]: F20.X-F29.X, F06.0-2, F10.5-F19.5) and affective syndromes (unipolar depression following ICD-10: F32.X, F33.X, F06.3; bipolar disorder following ICD-10: F30.X, F31X, F06.3) were clinically diagnosed by experienced psychiatrists and classified according to their predominant initial psychiatric syndromes. Preexisting or newly occurring neurological comorbidities were itemized but not considered as exclusion criteria whereas a comorbid diagnosis of dementia (ICD-10: F00.X-F04.X) led to exclusion. The clinical characteristics were extracted from the patients' discharge letters. The clinical findings and psychometric scores, including Clinical Global Impression (CGI), Global Assessment of Functioning (GAF), and psychopathological scores following the German Association for Methodology and Documentation in Psychiatry (AMDP), were extracted from the basic clinical documentation.

\section{Laboratory methods}

Paired CSF and serum samples were drawn at the same time following guideline recommendations [21]. The CSF and serum diagnostics were performed in the CSF laboratory of the Department of Neurology of the University Medical Center Freiburg (https://www.uniklinikfreiburg.de/neurologie/klinik/diagnostische-einrichtun gen/liquor-labor.html), and the CSF and serum samples were analyzed immediately after centrifugation. The basic quantitative protein diagnostics via nephelometry included total CSF protein concentration, serum/ CSF albumin, and serum/CSF IgG concentration (ProSpect System, Siemens, Erlangen, Germany). The CSF and serum albumin were used to calculate the $\mathrm{Q}_{\mathrm{Alb}}$ using the formula $\mathrm{Q}_{\mathrm{Alb}}=[\mathrm{Alb}] \mathrm{CSF} /[\mathrm{Alb}]$ serum $\times 1000$. The established age-dependent reference values for $\mathrm{Q}_{\mathrm{Alb}}$ were used: $<40$ years: $<6.5 \times 10^{-3} ; \quad 40-60$ years: $<8 \times 10^{-3}$; and $>60$ years: $<9.3 \times 10^{-3}$ [22]. In addition, the white blood cell (WBC) count and cytological staining were assessed by manual microscopy (Leica DMRB, Germany) using a Fuchs-Rosenthal counting chamber 
(Hecht-Assistant, Germany). All samples with elevated WBC counts were checked for blood contamination. In case of blood contamination the WBC counts were corrected according to the following formula: 1 cell $/ \mu \mathrm{l}$ of WBC count reduction per 1000 red blood cells/ $\mu$ l. Isoelectric focusing followed by immunofixation (Hydragel Isofocusing, Sebia, France) were used to determine oligoclonal bands (OCBs).

\section{Instrument-based diagnostics} Electroencephalography (EEG)

The admission procedures included an EEG examination in resting state and under hyperventilation $(\mathrm{HV})$. The evaluation was performed as part of clinical routine [20].

\section{Magnetic resonance imaging (MRI)}

At the least, the MRI protocol included T1-weighted (MPRAGE with isotropic $1 \mathrm{~mm}^{3}$ voxels on $3 \mathrm{~T}$ or axial $5 \mathrm{~mm}$ thick fast spin-echo slices on a $1.5 \mathrm{~T}$ scanner), FLAIR (3D SPACE sequence with isotropic $1 \mathrm{~mm}^{3}$ voxels on $3 \mathrm{~T}$ or coronal $3 \mathrm{~mm}$ thick fast spin-echo slices on a $1.5 \mathrm{~T}$ scanner), and DWI sequences (axial $5 \mathrm{~mm}$ thick slices). The evaluation was performed by experienced senior physicians in neuroradiology, and the MRI findings were classified as in the main study [20].

\section{Available data sets}

The initial study cohort included 992 patients [20], of which the $\mathrm{Q}_{\mathrm{Alb}}$ of 989 patients was available. Those 989 patients were analyzed in the current study. Due to the retrospective approach, some parameters are missing. The available data sets are presented in Table 1.

\section{Statistical analyses}

Statistical analysis was carried out using the Statistical Package for the Social Sciences (SPSS), version 24 (IBM Corp., Armonk, NY, USA). The results are mainly presented in a descriptive manner. Independent samples t-tests were conducted comparing age and dimensional variables between the subgroups of patients (without age difference). ANCOVA analyses were used to compare other dimensional variables (e.g., CSF $\mathrm{Q}_{\mathrm{Alb}}$ between female and male patients) between the subgroups corrected for age where indicated. The age-corrected $\mathrm{Q}_{\mathrm{Alb}}$ values were compared using $\mathrm{chi}^{2}$ tests. A binary logistic regression ("Wald statistics") was performed for agedependent categorical variables (e.g., the number of elevated CSF protein levels) between groups with age differences. The correlations between $\mathrm{Q}_{\mathrm{Alb}}$ levels and IRDA/ IRTA rates, clinical findings (number of suicide attempts and number of earlier inpatient stays), and psychometric scores (GAF, CGI, and AMDP) were analyzed using Spearman correlation. The correlation analyses were performed separately for female and male patients. A p-value of $<0.05$ was defined as statistically significant for $\mathrm{Q}_{\mathrm{Alb}}$ comparisons (the primary outcome parameter). This significance level was also used for further group comparisons and correlation analyses. Due to the exploratory approach of the additional statistical analyses, no correction for multiple testing was performed. Figure 1 was created using $\mathrm{R}$ [23].

\section{Results}

Description of the study population

The CSF of 545 female and 444 male patients was analyzed. The two subgroups differed significantly in age (females: $43.77 \pm 17.21$ years, males: $\quad 41.33 \pm 18.61$ years; $p=0.033)$. Detailed

Table 1 Overview of examined parameters of patients

\begin{tabular}{lll}
\hline & Parameters & Total N (female/male) \\
\hline Cerebrospinal fluid (CSF) markers & & \\
Signs of BCSFB dysfunction & Albumin quotients (AQ) & $989(545 / 444)$ \\
Further CSF basic analyses & Total protein & $988(544 / 444)$ \\
& White blood cell count & $979(536 / 443)$ \\
& lgG index & $989(545 / 444)$ \\
& OCBs in CSF & $965(529 / 436)$ \\
Instrument-based diagnostics & OCBs in serum & $964(529 / 435)$ \\
EEG & & $951(519 / 432)$ \\
MRI of the brain & Resting state & $802(441 / 361)$ \\
\hline
\end{tabular}

CSF, cerebrospinal fluid; BCSFB, blood-cerebrospinal fluid barrier; $A Q$, albumin quotient; lgG, immunoglobulin; OCB, oligoclonal bands; $E E G$, electroencephalography; $M R I$, magnetic resonance imaging 


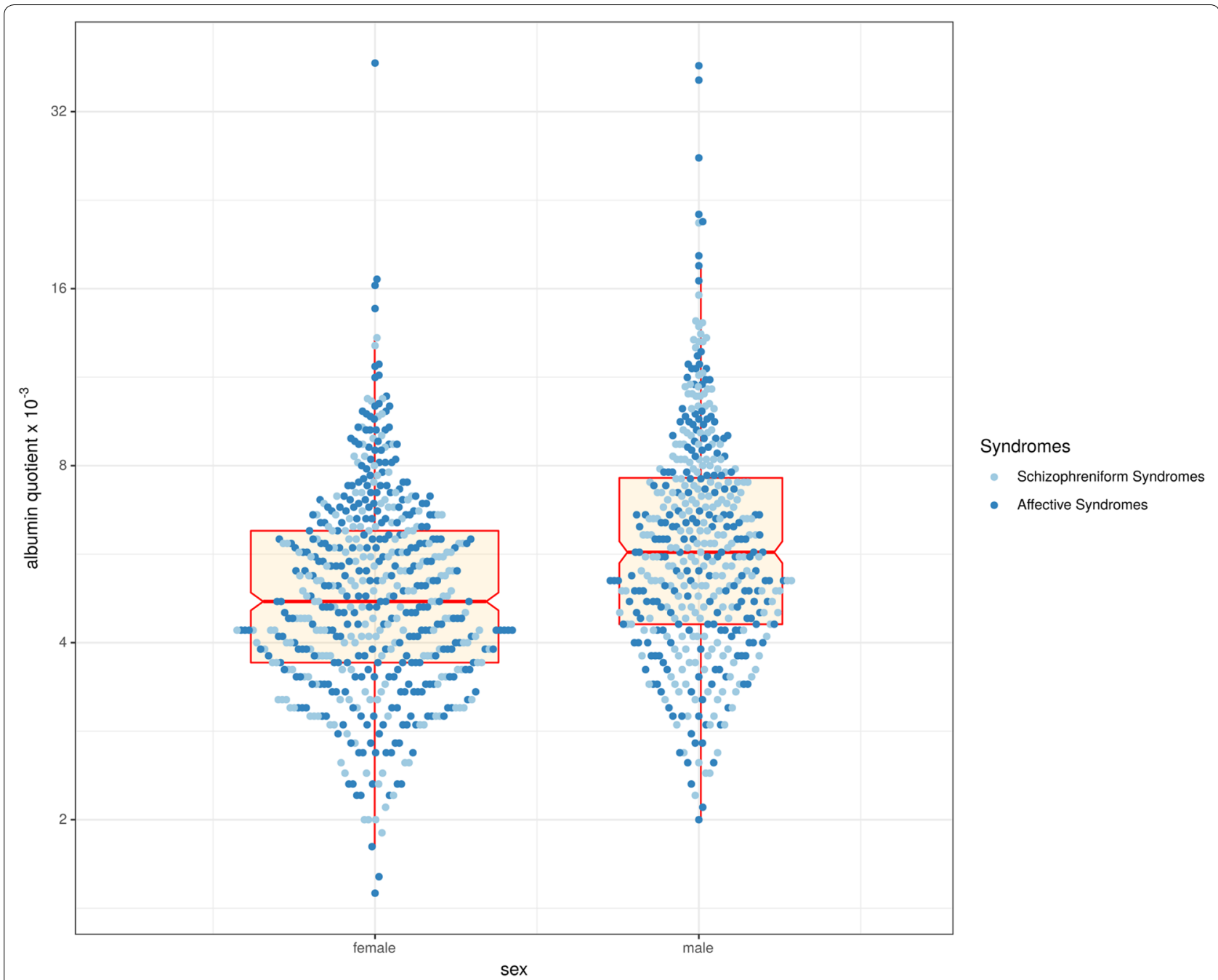

Fig. 1 Scatter plot diagram with levels of albumin quotients ( $y$-axis) and sex (x-axis). Single case data is shown. A box plot is added in red (quartiles and median of the distribution; the notches in the box sides give an approximation of the $95 \%$ confidence interval around the median). The data are presented on logarithmic scales

characterizations are presented in Table 2, and medications at the time of LP or upon admission are listed in Table 3.

\section{CSF-blood albumin quotients}

In total, $18 \%$ of all patients exhibited elevated $\mathrm{Q}_{\mathrm{Alb}}$ levels compared to age adjusted control levels [22]. Comparing male and female patients revealed a statistically significant difference $\left(\mathrm{chi}^{2}=42.625, \mathrm{p}<0.001\right)$, with a higher number of elevated age-corrected $\mathrm{Q}_{\mathrm{Alb}}$ in male patients $(26 \%)$ than in female patients $(10 \%)$. The mean values of the $\mathrm{Q}_{\mathrm{Alb}}$ also differed significantly $(\mathrm{F}=52.837, \quad \mathrm{CI}=[0.892 ; 1.676], \quad \mathrm{p}<0.001)$ between female $\left(\mathrm{M} \pm \mathrm{SD} \times 10^{-3}=5.23 \pm 2.56\right)$ and male patients $\left(\mathrm{M} \pm \mathrm{SD} \times 10^{-3}=6.52 \pm 3.69\right)$, as illustrated in Fig. 1 . This significant sex difference was found irrespective of clinical syndromes (Table 4).

\section{The effect of neurological comorbidity as a confounding factor on sex differences}

In order to rule out an effect of neurological comorbidities, a post hoc analysis was performed in the subgroup of patients without neurological comorbidities only. Female (age: $\mathrm{M}=42.39$ years, $\mathrm{SD}=16.42 ; \mathrm{N}=434$ ) and male (age: $\mathrm{M}=38.24$ years, $\mathrm{SD}=17.26 ; \mathrm{N}=354$ ) patients without neurological comorbidities $(\mathrm{N}=788,80 \%)$ differed significantly in age $(F=1.799, p=0.001)$. Significant differences 
Table 2 Description of the study population

\begin{tabular}{|c|c|c|c|}
\hline & \multicolumn{2}{|l|}{ All patients $(\mathrm{N}=989)$} & \multirow[t]{2}{*}{ Statistics } \\
\hline & Females $(\mathrm{N}=545 ; 55 \%)$ & Males $(\mathrm{N}=444 ; 45 \%)$ & \\
\hline Age (range) in years $\pm S D$ & $43.77 \pm 17.21$ (from 18-90) & $41.33 \pm 18.61$ (from 18-85) & $\begin{array}{l}F=8.183 \\
p=0.033\end{array}$ \\
\hline \multicolumn{4}{|l|}{ Syndrome } \\
\hline Schizophreniform syndrome & $N=248(46 \%)$ & $N=208(47 \%)$ & \\
\hline \multicolumn{4}{|l|}{ Affective syndrome } \\
\hline Depressive syndrome & $N=249(46 \%)$ & $N=203(46 \%)$ & \\
\hline Bipolar syndrome & $N=48(9 \%)$ & $N=33(7 \%)$ & \\
\hline \multicolumn{4}{|l|}{ Clinical course } \\
\hline First episode & $143(27 \%)$ & $135(30 \%)$ & \\
\hline Chronic (> 2 years) & $133(25 \%)$ & $125(28 \%)$ & \\
\hline Recurrent & $261(49 \%)$ & $183(41 \%)$ & \\
\hline Unknown & $8(1 \%)$ & $1(0.2 \%)$ & \\
\hline \multicolumn{4}{|c|}{ Previous/current comorbid psychiatric disorders } \\
\hline Neurodevelopmental disorders ${ }^{\mathrm{a}}$ & $38(7 \%)$ & $51(11 \%)$ & \\
\hline Personality disorders & $26(5 \%)$ & $17(4 \%)$ & \\
\hline Substance abuse/dependence & $50(9 \%)$ & $63(14 \%)$ & \\
\hline Anxiety disorders & $17(3 \%)$ & $16(4 \%)$ & \\
\hline OCD & $17(3 \%)$ & $7(2 \%)$ & \\
\hline PTSD & $12(2 \%)$ & $6(1 \%)$ & \\
\hline Cognitive disorders (MCl) & $15(3 \%)$ & $26(6 \%)$ & \\
\hline Sleep disturbances & $9(2 \%)$ & $9(2 \%)$ & \\
\hline Eating disorders & $16(3 \%)$ & $0(0 \%)$ & \\
\hline Somatoform disorder ${ }^{b}$ & $20(4 \%)$ & $12(3 \%)$ & \\
\hline Others ${ }^{c}$ & $7(1 \%)$ & $2(0.5 \%)$ & \\
\hline \multicolumn{4}{|c|}{ Previous/current comorbid neurological disorders } \\
\hline Neurovascular & $10(2 \%)$ & $14(3 \%)$ & \\
\hline Demyelinating $^{d}$ & $2(0.4 \%)$ & $1(0.2 \%)$ & \\
\hline Extrapyramidal/movement & $3(0.6 \%)$ & $7(2 \%)$ & \\
\hline Infectious & $3(0.6 \%)$ & $3(0.7 \%)$ & \\
\hline Tumors & $2(0.4 \%)$ & $1(0.2 \%)$ & \\
\hline Paroxysmal & $12(2 \%)$ & $7(2 \%)$ & \\
\hline Traumatic injuries & $11(2 \%)$ & $12(3 \%)$ & \\
\hline Polyneuropathy & $7(1 \%)$ & $13(3 \%)$ & \\
\hline Migraine and other headaches & $36(7 \%)$ & $10(2 \%)$ & \\
\hline Restless Legs Syndrome & $9(2 \%)$ & $7(2 \%)$ & \\
\hline Hydrocephalus & $4(0.7 \%)$ & $5(1 \%)$ & \\
\hline Others & $12(2 \%)$ & $10(2 \%)$ & \\
\hline \multicolumn{4}{|l|}{ School education } \\
\hline No degree & $9(2 \%)$ & $5(1 \%)$ & \\
\hline Low degree & $87(20 \%)$ & $86(23 \%)$ & \\
\hline Medium degree & 137 (31\%) & $97(26 \%)$ & \\
\hline High degree & 201 (46\%) & $182(49 \%)$ & \\
\hline Other & $3(0.7 \%)$ & $5(1 \%)$ & \\
\hline Unknown & 108 (20\%) & $69(16 \%)$ & \\
\hline
\end{tabular}

$(\mathrm{F}=36.029, \mathrm{p}<0.001)$ in levels of $\mathrm{Q}_{\mathrm{Alb}}$ were discerned, with higher rates in male $\left(\mathrm{M} \pm \mathrm{SD} \times 10^{-3}=6.21 \pm 3.41\right)$ than in female patients $\left(\mathrm{M} \pm \mathrm{SD} \times 10^{-3}=5.18 \pm 2.57\right)$ in the agecorrected ANCOVA analysis. 
Table 2 (continued)

\begin{tabular}{|c|c|c|c|}
\hline & \multicolumn{2}{|l|}{ All patients $(\mathrm{N}=989)$} & \multirow[t]{2}{*}{ Statistics } \\
\hline & Females $(\mathrm{N}=545 ; 55 \%)$ & Males $(\mathrm{N}=444 ; 45 \%)$ & \\
\hline \multicolumn{4}{|l|}{ Occupation } \\
\hline Employed & $150(33 \%)$ & $114(30 \%)$ & \\
\hline House-wife/-husband & $28(6 \%)$ & $0(0 \%)$ & \\
\hline Unemployed & $59(13 \%)$ & $67(18 \%)$ & \\
\hline Disability pension & $56(12 \%)$ & $45(12 \%)$ & \\
\hline Retirement pension & $72(16 \%)$ & $59(16 \%)$ & \\
\hline In-training/in studies/retraining & $68(15 \%)$ & $81(21 \%)$ & \\
\hline Others & $18(4 \%)$ & $10(3 \%)$ & \\
\hline Unknown & $94(17 \%)$ & $67(15 \%)$ & \\
\hline
\end{tabular}

SD, standard deviation; ADHD, attention deficit hyperactivity disorder; PTSD, post-traumatic stress disorder; $O C D$, obsessive-compulsive disorder; $M C l$, mild cognitive impairment

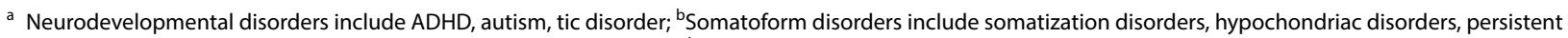
pain disorders. ${ }^{\circ}$ Other psychiatric comorbidity: Dissociative disorders. ${ }^{\mathrm{d}}$ Relapse of multiple sclerosis with pure psychiatric manifestations. Multiple sclerosis has been initially diagnosed

Table 3 Psychopharmacological medication

\begin{tabular}{|c|c|c|c|}
\hline & \multicolumn{2}{|l|}{ All patients $(\mathrm{N}=989)$} & \multirow[t]{2}{*}{ Statistics } \\
\hline & Females $(\mathrm{N}=545 ; 55 \%)$ & Males $(\mathrm{N}=444 ; 45 \%)$ & \\
\hline \multicolumn{4}{|c|}{ Overall psychopharmacological treatment } \\
\hline Yes & $498(94 \%)$ & $415(95 \%)$ & \multirow{3}{*}{$\begin{array}{c}\mathrm{Chi}^{2}=0.811 \\
p=0.368\end{array}$} \\
\hline No & $34(6 \%)$ & $22(5 \%)$ & \\
\hline Unknown & $13(1 \%)$ & $7(2 \%)$ & \\
\hline \multicolumn{4}{|l|}{ Antidepressants } \\
\hline Overall & $295(59 \%)$ & $221(53 \%)$ & \\
\hline Tricyclic & $31(11 \%)$ & $28(13 \%)$ & \\
\hline SSRI, SNRI, NDRI, NARI & $261(88 \%)$ & $188(85 \%)$ & \\
\hline MAO inhibitors & $3(1 \%)$ & $5(2 \%)$ & \\
\hline \multicolumn{4}{|l|}{ Antipsychotics } \\
\hline Overall & $384(77 \%)$ & $286(69 \%)$ & \\
\hline "Typical" & $83(17 \%)$ & $59(14 \%)$ & \\
\hline Low-potency & $61(73 \%)$ & $39(66 \%)$ & \\
\hline Medium-potency & $0(0 \%)$ & $0(0 \%)$ & \\
\hline High-potency & $22(27 \%)$ & $20(34 \%)$ & \\
\hline "Atypical" & $356(71 \%)$ & $263(63 \%)$ & \\
\hline \multicolumn{4}{|l|}{ Mood stabilizers } \\
\hline Lithium & $92(18 \%)$ & $57(14 \%)$ & \\
\hline Anticonvulsants & $85(17 \%)$ & $58(14 \%)$ & \\
\hline Benzodiazepines & $87(17 \%)$ & $58(14 \%)$ & \\
\hline \multicolumn{4}{|c|}{ Number of different medication classes per patient } \\
\hline Same class/only one drug & $174(35 \%)$ & $202(49 \%)$ & \\
\hline Two drugs & $189(38 \%)$ & $135(33 \%)$ & \\
\hline Three drugs & $97(19 \%)$ & $68(16 \%)$ & \\
\hline Four drugs & $35(7 \%)$ & $10(2 \%)$ & \\
\hline Five drugs & $3(0.6 \%)$ & $0(0 \%)$ & \\
\hline
\end{tabular}

SSRI, selective-serotonin-reuptake-inhibitor; SNRI, serotonin-noradrenalin-reuptake-inhibitor; NDRI, norepinephrine-dopamine-reuptake-inhibitor; NARI, noradrenalin-reuptake-inhibitor; MAO, monoamine oxidase 
Table 4 Signs of blood-cerebrospinal fluid barrier dysfunction: albumin quotients and numbers for female and male patients in relation to reference values

\begin{tabular}{|c|c|c|c|c|c|}
\hline & Reference & All patient $(\mathrm{N}=989)$ & Females $(\mathrm{N}=545 ; 55 \%)$ & Males ( $\mathrm{N}=444 ; 45 \%)$ & Statistics \\
\hline \multicolumn{6}{|l|}{ All patients } \\
\hline $\begin{array}{l}\text { Albumin quotients } \\
\left(\times 10^{-3}, \text { Mean } \pm S D\right. \\
\text { median and range })\end{array}$ & & $\begin{array}{l}5.81 \pm 3.185 .10 \\
(1.50-38.70)\end{array}$ & $\begin{array}{l}5.23 \pm 2.56 \\
4.70(1.50-38.70)\end{array}$ & $\begin{array}{l}6.52 \pm 3.69 \\
5.70(2.00-38.30)\end{array}$ & $\begin{array}{l}F=52.837 \\
C l=[0.892 ; 1.676] \\
p<0.001\end{array}$ \\
\hline \multirow[t]{2}{*}{$\begin{array}{l}\text { Increased albumin } \\
\text { quotients, numbers } \\
\text { of patient }\end{array}$} & $\begin{array}{l}<40 y::<6.5 \times 10^{-3} \\
40-60 y::<8 \times 10^{3} \\
>60 y::<9.3 \times 10^{-3}\end{array}$ & $\begin{array}{l}\uparrow: 174(18 \%) \\
\leftrightarrow: 815(82 \%)\end{array}$ & $\begin{array}{l}\uparrow: 57(10 \%) \\
\leftrightarrow: 488(90 \%)\end{array}$ & $\begin{array}{l}\uparrow: 117(26 \%) \\
\leftrightarrow: 327(74 \%)\end{array}$ & $\begin{array}{l}\mathrm{Chi}^{2}=42.625 \\
\mathrm{p}<0.001\end{array}$ \\
\hline & \multicolumn{2}{|c|}{ Schizophreniform syndrome $(\mathrm{N}=456)$} & Females $(\mathrm{N}=248 ; 54 \%)$ & Males $(\mathrm{N}=208 ; 46 \%)$ & \\
\hline $\begin{array}{l}\text { Albumin quotients } \\
\left(\times 10^{-3}, \text { Mean } \pm S D\right)\end{array}$ & & $5.64 \pm 3.18$ & $5.29 \pm 3.02$ & $6.06 \pm 3.32$ & $\begin{array}{l}F=11.906 \\
C l=[0.187 ; 1.354] \\
p=0.001\end{array}$ \\
\hline \multirow[t]{2}{*}{$\begin{array}{l}\text { Increased albumin } \\
\text { quotients, numbers of } \\
\text { patients }\end{array}$} & $\begin{array}{l}<40 y::<6.5 \times 10^{-3} \\
40-60 y::<8 \times 10^{-} \\
{ }^{3}>60 y::<9.3 \times 10^{-3}\end{array}$ & $\begin{array}{l}\uparrow: 85(19 \%) \\
\leftrightarrow: 371(81 \%)\end{array}$ & $\begin{array}{l}\uparrow: 33(13 \%) \\
\leftrightarrow: 215(87 \%)\end{array}$ & $\begin{array}{l}\uparrow: 52(25 \%) \\
\leftrightarrow: 156(75 \%)\end{array}$ & $\begin{array}{l}\mathrm{Chi}^{2}=10.200 \\
\mathrm{p}=0.001\end{array}$ \\
\hline & \multicolumn{2}{|c|}{ Affective syndrome $(\mathrm{N}=533)$} & Females $(\mathrm{N}=297,56 \%)$ & Males $(\mathrm{N}=236,44 \%)$ & \\
\hline $\begin{array}{l}\text { Albumin quotients } \\
\left(\times 10^{-3}, \text { Mean } \pm S D\right)\end{array}$ & & $5.95 \pm 3.19$ & $5.19 \pm 2.11$ & $6.92 \pm 3.96$ & $\begin{array}{l}F=27.991 \\
C l=[1.208 ; 2.260] \\
p<0.001\end{array}$ \\
\hline $\begin{array}{l}\text { Increased albumin } \\
\text { quotients, numbers of } \\
\text { patients }\end{array}$ & 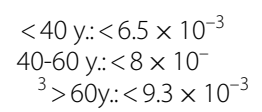 & $\begin{array}{l}\uparrow: 89(17 \%) \\
\leftrightarrow: 444(83 \%)\end{array}$ & $\begin{array}{l}\uparrow: 24(8 \%) \\
\leftrightarrow: 273(92 \%)\end{array}$ & $\begin{array}{l}\uparrow: 65(28 \%) \\
\leftrightarrow: 171(72 \%)\end{array}$ & $\begin{array}{l}\mathrm{Chi}^{2}=35.808 \\
\mathrm{p}<0.001\end{array}$ \\
\hline
\end{tabular}

$\mathrm{SD}$, standard deviation; $\mathrm{Cl}$, confidence interval; $\uparrow$, increased; $\leftrightarrow$, in normal range

\section{Cerebrospinal fluid basic parameters and instrument-based diagnostics}

The CSF protein levels were elevated in male as compared to female patients $(\beta=0.859$, Wald $=39.542$, $\mathrm{p}<0.001)$. Overall, male patients exhibited some degree of CSF alteration significantly more frequently than female patients $(\beta=0.747$, Wald $=30.608, \mathrm{p}<0.001)$. There were no significant sex differences in white blood cell counts, the IgG index, or oligoclonal bands (Table 5). EEG analyses revealed no significant sex differences. Female patients more frequently had inflammatory changes in the MRI (Wald $=4.360, \mathrm{p}=0.037$, Table 6).

\section{Correlation analyses \\ Female patients}

The $\mathrm{Q}_{\mathrm{Alb}}$ correlated significantly with the AMDP scores for fears and compulsions $(r=-0.161, p=0.001 ; N=422)$, and the AMDP scores for delusions $(\mathrm{r}=-0.114, \mathrm{p}=0.019$; $\mathrm{N}=421$ ).

\section{Male patients}

The $\mathrm{Q}_{\mathrm{Alb}}$ were significantly correlated with CGI score on admission $(\mathrm{r}=0.168, \mathrm{p}=0.001 ; \mathrm{N}=388)$, number of earlier suicide attempts $(\mathrm{r}=0.213, \mathrm{p}=0.003 ; \mathrm{N}=197)$, and the AMDP scores for fears and compulsions $(\mathrm{r}=-0.161, \mathrm{p}=0.003 ; \mathrm{N}=352)$.

\section{Discussion}

The main result of this study is a significantly elevated $\mathrm{Q}_{\mathrm{Alb}}$ in men compared to women, which at first glance and in accordance with traditional interpretations suggests that the integrity of the $\mathrm{BBB}$ is more often impaired in men than in women with schizophrenic and affective psychosis. However, due to current recognition that there are three or more brain barriers and interfaces [8-11], $Q_{\mathrm{Alb}}$ is now considered a measure of the overall BCSFB $[12,13,15]$. $\mathrm{Q}_{\mathrm{Alb}}$ represents a measure of predominantly blood-derived albumin within the CSF in relation to blood albumin concentration. The CSF albumin content determined from lumbar CSF, can potentially be influenced by factors other than plasma concentration. The exchange of CSF and its content with the tissues surrounding the brain ventricles and subarachnoid spaces could alter the composition. Also, variable CSF flow dynamics passing through 
Table 5 Cerebrospinal fluid (CSF) basic parameters

\begin{tabular}{|c|c|c|c|c|c|c|}
\hline & Reference & All patient $(\mathrm{N}=989)$ & & $\begin{array}{l}\text { Females ( } N=545 \text {; } \\
55 \%)\end{array}$ & Males $(\mathrm{N}=444 ; 45 \%)$ & Statistics \\
\hline \multicolumn{7}{|c|}{ Cerebrospinal fluid (CSF) basic parameters } \\
\hline $\begin{array}{l}\text { CSF protein concentra- } \\
\text { tion (Mean } \pm S D \\
\text { range) }\end{array}$ & & $\begin{array}{l}470.91 \pm 236.45 \text { (from } \\
107 \text { to } 2890 \mathrm{mg} / \mathrm{l})\end{array}$ & $423.74 \pm 196.84$ & & $528.70 \pm 266.44$ & $\begin{array}{l}F=60.366 \\
p<0.001\end{array}$ \\
\hline $\begin{array}{l}\text { Increased CSF protein } \\
\text { concentration }\end{array}$ & $<450 \mathrm{mg} / \mathrm{l}$ & $\begin{array}{l}\uparrow: 447(45 \%) \\
\leftrightarrow: 541(55 \%) \\
\text { n.a.: } 1(0.1 \%)\end{array}$ & $\begin{array}{l}\uparrow: 202(37 \%) \\
\leftrightarrow: 342(63 \%) \\
\text { n.a.: } 1(0.2 \%)\end{array}$ & & $\begin{array}{l}\uparrow: 245(55 \%) \\
\leftrightarrow: 199(45 \%) \\
\text { n.a.: } 0(0 \%)\end{array}$ & $\begin{array}{l}\beta=0.859 \\
\text { Wald }=39.542 \\
p<0.001\end{array}$ \\
\hline $\begin{array}{l}\text { WBC counts } \\
\quad(\text { Mean } \pm \text { SD })\end{array}$ & & $\begin{array}{l}1.96 \pm 4.85 \text { (from } 1 \text { to } \\
101 / \mu \mathrm{l})\end{array}$ & $1.99 \pm 5.33$ & & $1.93 \pm 4.20$ & $\begin{array}{l}F=0.070 \\
P=0.791\end{array}$ \\
\hline Increased WBC counts & $<5 / \mu l$ & $\begin{array}{l}\uparrow: 37(4 \%) \\
\leftrightarrow: 942(96 \%) \\
\text { n.a.: } 1(0.1 \%)\end{array}$ & $\begin{array}{l}\uparrow: 25(5 \%) \\
\leftrightarrow: 511(95 \%) \\
\text { n.a.: } 9(2 \%)\end{array}$ & & $\begin{array}{l}\uparrow: 12(3 \%) \\
\leftrightarrow: 431(97 \%) \\
\text { n.a.: } 1(0.2 \%)\end{array}$ & $\begin{array}{l}\text { Wald }=2.777 \\
p=0.096\end{array}$ \\
\hline IgG-Index (Mean \pm SD) & & $0.50 \pm 0.10$ & $0.50 \pm 0.11$ & & $0.50 \pm 0.09$ & $\begin{array}{l}F=0.508 \\
p=0.476\end{array}$ \\
\hline Increased lgG indices & & $\begin{array}{l}\uparrow: 19(2 \%) \\
\leftrightarrow: 970(98 \%)\end{array}$ & $\begin{array}{l}\uparrow: 14(3 \%) \\
\leftrightarrow: 531(97 \%)\end{array}$ & & $\begin{array}{l}\uparrow: 5(1 \%) \\
\leftrightarrow: 439(99 \%)\end{array}$ & $\begin{array}{l}\text { Wald }=2.367 \\
p=0.124\end{array}$ \\
\hline $\begin{array}{l}\text { Isolated OCB in CSF } \\
\text { OCBs in CSF and Serum }\end{array}$ & $\begin{array}{l}\text { negative } \\
\text { negative }\end{array}$ & $\begin{array}{l}40(4 \%) \\
\text { n.a.: } 24(2 \%) \\
52(5 \%) \\
\text { n.a.: } 25(3 \%)\end{array}$ & $\begin{array}{l}21(4 \%) \\
\text { n.a.: } 16(3 \%) \\
27(5 \%) \\
\text { n.a.: } 16(3 \%)\end{array}$ & & $\begin{array}{l}19(4 \%) \\
\text { n.a.: } 8(2 \%) \\
25(6 \%) \\
\text { n.а.: } 9(2 \%)\end{array}$ & $\begin{array}{l}\text { Wald }=0.095 \\
p=0.758 \\
\text { Wald }=0.456 \\
p=0.500\end{array}$ \\
\hline $\begin{array}{l}\text { Inflammatory CSF } \\
\text { changes }^{\mathrm{a}}\end{array}$ & & 77 / 989 (8\%) & 48 / 545 (9\%) & & $29 / 444(7 \%)$ & $\begin{array}{l}\text { Wald }=1.754 \\
p=0.185\end{array}$ \\
\hline $\begin{array}{l}\text { Overall Basic CSF } \\
\text { changes }^{\text {b }}\end{array}$ & & $491 / 989$ (50\%) & $232 / 545(43 \%)$ & & $259 / 444(58 \%)$ & $\begin{array}{l}\beta=0.747 \\
\text { Wald }=30.608 \\
p<0.001\end{array}$ \\
\hline
\end{tabular}

WBC, white blood cell; OCBs, oligoclonal bands; CSF, cerebrospinal fluid; SD, standard deviation; $\uparrow$, increased; $\leftrightarrow$, in normal range; n.a., not available

a Inflammatory CSF changes: WBC counts increased and/or lgG indices increased and/or CSF specific oligoclonal bands. ${ }^{b}$ Overall basic CSF alterations: Inflammatory CSF changes and/or increased albumin quotients and/or increased protein concentrations

the subarachnoid spaces to the lumbar puncture site, which lies further down the spine, appears to have an influence.

\section{Integration of our results into the context of current studies}

This finding is consistent with the results of two samples of patients with neurological disorders, including some healthy controls $[18,19]$. In a first large study including 27,263 patients with neurological diseases, elevated $\mathrm{Q}_{\mathrm{Alb}}$ values were observed more frequently in male patients [19]. However, details about the syndromes, diagnostic findings, and medication were not available. The study also included data from 335 healthy controls (although the definition of "healthy" was not specified), who displayed the same sex differences as well [19]. A similar study including 1209 patients with different neurological disorders also reported similar sex differences [18]. In the present study, these findings could be confirmed in a more homogeneous, well-characterized psychiatric cohort including diagnostic findings and information about potential confounding factors, such as comorbidity or psychopharmacological medication. Another study investigating a large cohort of 1,079,193 participants with unknown characteristics evaluated serum albumin and found sex differences depending on age [24]. In the current study, protein concentrations were also higher in male than in female patients, similar to previous studies $[25,26]$. Overall, the sex difference in $\mathrm{Q}_{\mathrm{Alb}}$ levels appears to be independent of neurological or psychiatric diagnoses, which therefore strongly suggests bias from some general factors associated with sex, which remain to be elucidated.

\section{Possible explanations for the sex differences in albumin quotients}

Sex-related differences in body height, specifically spine length, may constitute a plausible supporting factor for this bias, given that sex appears to be predominantly associated with a systematic difference in average height. Since body height was unfortunately not recorded in the present cohort, the average heights of the German population were used as an approximate comparison. Measures for the German population from a microcensus of 2017 reported a mean height for ages 18-40 of $181 \mathrm{~cm}$ for males and of $167 \mathrm{~cm}$ for females, and for ages $40-65,179 \mathrm{~cm}$ for males and $166 \mathrm{~cm}$ for females, respectively (Statistisches Bundesamt, https://www.destatis. 
Table 6 Instrument based diagnostics

\begin{tabular}{|c|c|c|c|c|}
\hline & All patients $(\mathrm{N}=989)$ & Females $(\mathrm{N}=545 ; 55 \%)$ & Males $(\mathrm{N}=444 ; 45 \%)$ & Statistics \\
\hline EEG Alterations & $N=951(96 \%)$ & $N=519(95 \%)$ & $N=432(97 \%)$ & \\
\hline Continuous generalized slow activity & $34(4 \%)$ & $18(3 \%)$ & $16(4 \%)$ & Wald $=0.105 p=0.746$ \\
\hline Continuous regional slow activity & $6(0.6 \%)$ & $1(0.2 \%)$ & $5(1 \%)$ & Wald $=2.748 p=0.097$ \\
\hline Intermittent generalized slow activity & $161(17 \%)$ & $95(18 \%)$ & $66(15 \%)$ & Wald $=1.789 p=0.181$ \\
\hline Intermittent regional slow activity & $52(5 \%)$ & $31(6 \%)$ & $21(5 \%)$ & Wald $=0.462 p=0.497$ \\
\hline Epileptic pattern & $29(3 \%)$ & $15(3 \%)$ & $14(3 \%)$ & Wald $=0.003 p=0.958$ \\
\hline EEG overall alterations & $240 / 951(25 \%)$ & $136 / 519$ (26\%) & 104 / 432 (24\%) & Wald $=0.641 p=0.423$ \\
\hline MRI Alterations & $N=894(90 \%)$ & $N=498(91 \%)$ & $N=396(89 \%)$ & \\
\hline White/Grey matter changes overalla & $460 / 894(51 \%)$ & $269 / 498(54 \%)$ & 191 / 396 (48\%) & Wald $=0.906 p=0.341$ \\
\hline Non-specific white matter changes & $374(42 \%)$ & $212(43 \%)$ & $162(41 \%)$ & Wald $=0.042 p=0.837$ \\
\hline $\begin{array}{l}\text { Grey matter changes of amygdalae, hippocampi, } \\
\text { other limbic structures }\end{array}$ & $12(1 \%)$ & $8(2 \%)$ & $4(1 \%)$ & Wald $=0.400 p=0.527$ \\
\hline $\begin{array}{l}\text { Possible/probable/ definite (post-) inflammatory } \\
\text { changes }\end{array}$ & $77(9 \%)$ & $52(10 \%)$ & $25(6 \%)$ & Wald $=4.360 p=0.037$ \\
\hline Atrophic changes ${ }^{\mathrm{b}}$ & $107(12 \%)$ & $52(10 \%)$ & $55(14 \%)$ & Wald $=3.702 p=0.054$ \\
\hline $\begin{array}{l}\text { Macroangiopathic vascular alterations (post- } \\
\text { ischemic changes) }\end{array}$ & $33(4 \%)$ & $17(3 \%)$ & $16(4 \%)$ & Wald $=0.306 p=0.580$ \\
\hline Microhemorrhage & $16(2 \%)$ & $10(2 \%)$ & $6(2 \%)$ & Wald $=0.244 p=0.621$ \\
\hline Cysts & $118(13 \%)$ & $67(13 \%)$ & $51(13 \%)$ & Wald $=0.213 p=0.644$ \\
\hline Tumors & $13(1 \%)$ & $9(2 \%)$ & $4(1 \%)$ & Wald $=0.834 p=0.361$ \\
\hline Anatomical variants and other changes & $208(23 \%)$ & $118(24 \%)$ & $90(23 \%)$ & Wald $=0.156 p=0.693$ \\
\hline Overall MRI changes & $639 / 894(71 \%)$ & $366 / 498(73 \%)$ & 273 / $396(69 \%)$ & Wald $=0.531 p=0.466$ \\
\hline
\end{tabular}

Several EEG and MRI changes were noted, if existing.

EEG, electroencephalography; IRDA/IRTA, intermittent rhythmic generalized delta/theta activity; MRI, magnetic resonance imaging

a White/grey matter changes overall: non-specific white matter changes and/or gray matter changes of amygdalae, hippocampi, other limbic structures and/or (post-) inflammatory changes. ${ }^{b}$ Atrophic changes overall: generalized cortical atrophy and/or localized atrophy and/or ventricle enlargement

de). Thus, extrapolating from a general German population broadly comparable to our patient group (female mean age 43.77 years; male mean age 41.33 years) indicates a systematic height difference between the sexes by approximately $13.5 \mathrm{~cm}$. This height difference implies an analogous difference of spine length between the sexes, although leg length might represent a confounder. The assumed sex differences in body height of $>10 \mathrm{~cm}$ and, as a consequence, in spine length between men and women, would be able to explain sex-related increases of $\mathrm{Q}_{\mathrm{Alb}}$ in males as compared to females because of the longer CSF flow distance down the spinal cord in men and a comparably shorter CSF flow distance in women because of shorter female spine size in related subarachnoid spaces. This explanatory approach is in line with replicated findings of a rostro-caudal gradient of $\mathrm{Q}_{\mathrm{Alb}}$ with increasing $Q_{\text {Alb }}$ values in CSF taken at sites down the neuraxis from the site of production in the ventricles to the most distal sites [14, 15, 27]. It also agrees in principle with a decrease in $\mathrm{Q}_{\mathrm{Alb}}$ when CSF was taken in sequential portioned volumes at the lumbar site $[15,28]$, which led to the general recommendation to always take fixed total volumes of CSF for examination to avoid this volume bias in routine diagnostics $[12,13,21]$. In addition, the suggested explanation matches with the flow-diffusionmodel of BCSFB $[14,15]$. The slow net flow of CSF down the neuraxis within the subarachnoid spaces is apparently related to and determined by the slow outflow of CSF along spinal nerves $[29,30]$, first described by Quincke in 1872 but widely forgotten until recently $[31,32]$.

Further influences could be caused by differences in body mass index (BMI), hormonal factors, and other sexrelated factors. Although BMI was also not assessed in the present patient cohort, we expect a similar distribution in our patient population as BMI distribution was similar between the sexes in the general German population (ages 18-40: 25.4 for males vs. 23.4 for females; ages 40-65: 27.3 for males vs. 25.3 for females; Statistisches Bundesamt, https://www.destatis.de). Regarding hormonal factors, it is for example known that estrogens may provide parallel neuroprotective benefits on the stability of the $\mathrm{BBB} / \mathrm{BCSFB}$ by preventing tight-junction breakdown through the up-regulation of anti-inflammatory annexin 1 (ANXA1) expression and by limiting lymphocyte migration through the modulation of the endothelial intercellular adhesion molecule 1 (ICAM-1) [33], which 
could indeed contribute to a better functioning of the $\mathrm{BBB} / \mathrm{BCSFB}$ in females. Other hypothetical causes for the observed sex differences could include a sex-specific expression of secretion molecules, tight junctions or endothelial cells, but also sex-differences in the number of patients with consumption of alcohol or (illegal) drugs in the past.

\section{Clinical consequences}

Irrespective of the cause, the $\mathrm{Q}_{\mathrm{Alb}}$ findings should be corrected for sex ratio in future CSF studies in psychiatric and neurological patients. In addition to the established age-dependent correction, the present results suggest adapting or stratifying the reference values of $\mathrm{Q}_{\mathrm{Alb}}$ for sex. If our considerations regarding body height are confirmed in future studies, a correction based on body size or spine length would be preferable. Nevertheless, it should be recognized that even with corrected $\mathrm{Q}_{\mathrm{Alb}}$ a relevant subgroup with $\mathrm{BBB} / \mathrm{BCSFB}$ dysfunction will persist, which cannot be explained by the bias and thus may relate to yet undefined brain pathologies as suggested from findings of abnormal $\mathrm{Q}_{\mathrm{Alb}}$ in various psychiatric and neurological disorders [20,34-39]. Additionally, it should be noted that a number of other CSF abnormalities have been reported in psychiatric patient cohorts [20, 38, 40-46]. Progress in this research area for psychiatry will require new and more sensitive methods of CSF analysis and an increased understanding of the complex interactions of biochemical and mechanistic influences on the dynamics of CSF contents under normal and pathological conditions.

\section{Limitations}

The limitation of the present study is its retrospective, open, and uncontrolled design. Patients with affective syndromes received an LP only in selected cases because the diagnostic approach has evolved through the years [20, 43-45]. Therefore, the sex differences are not representative of all patients with affective syndromes. Due to the open design, patients with neurological comorbidity were also included. Therefore, BBB/BCSFB dysfunctions could, theoretically, also be caused by pathophysiology related to comorbid conditions. However, the $\mathrm{Q}_{\mathrm{Alb}}$ values remained significantly different between sexes even when restricting analyses to patients without neurological comorbidity. Examinations of large, healthy control groups are lacking so far, so the present findings cannot be generalized to neurologically and/or mentally healthy populations because LPs of a large group of healthy volunteers is difficult to justify on ethical grounds. In contrast to previous studies $[18,19]$, the authors examined a more homogenous, well-characterized group of psychiatric patients. Another limitation is that the $\mathrm{Q}_{\mathrm{Alb}}$ levels were not corrected for blood admixture and this could have influenced the results; however, this effect would apply equally to female and male patients. Unfortunately, the influences of other possible contributing factors, such as body weight, alcohol/nicotine consumption, or diabetes mellitus on BBB/BCSFB function, could not be analyzed in the present study, and body height was only estimated based on the mean heights of the current German population. In addition, exact measures of spine length or suboccipital-to-puncture-site distance for our physiological explanation of the bias were not available. A correction for the potential influence of psychotropic drugs was not performed, because equal numbers of male and female patients had been treated.

\section{Conclusions}

The current study reveals a significant sex difference in $\mathrm{Q}_{\mathrm{Alb}}$ levels, with higher levels in males than in females. The present results confirm and extend previous findings of a systematic sex difference in $\mathrm{Q}_{\mathrm{Alb}}$, which exists not only in neurological patient cohorts but also in psychiatric patients, independent of diagnosis. Irrespective of the underlying pathophysiology, which remains to be elucidated, the present findings suggest the need for an extended correction mode of $\mathrm{Q}_{\mathrm{Alb}}$ reference values in the future.

\section{Acknowledgements \\ DS and DE were funded by the Berta-Ottenstein-Programme for Advanced Clinician Scientists, Faculty of Medicine, University of Freiburg.}

\section{Authors' contributions}

$\mathrm{SMe}, \mathrm{KB}$, and DE wrote the paper. $\mathrm{LTVE}$, SMe, and DE created the study design. RD performed and interpreted the CSF basic analyses. BF supported EEG analyses and statistical analyses. SMa created Fig. 1 and supported statistical analyses. KB worked out the interpretation about the sex bias by CSF flow principles and beyond. $\mathrm{HU}$ was responsible for MRI analyses and interpretation. MAS, KR, DD, KN, DS, HU, HP, KD and LTVE supported the clinical interpretation and critically revised the manuscript. All authors were critically involved in the theoretical discussion and composition of the manuscript. All authors read and approved the final manuscript.

\section{Funding}

Open Access funding enabled and organized by Projekt DEAL. The article processing charge was funded by the Baden-Wuerttemberg Ministry of Science, Research and Art and the University of Freiburg in the funding programme Open Access Publishing

\section{Availability of data and materials}

All necessary information is displayed descriptively in the results section.

\section{Ethics approval and consent to participate}

This retrospective analysis received approval from the local ethics committee of the University of Freiburg (EK Fr 396/18). The patients gave their written informed consent before the lumbar puncture.

\section{Consent for publication}

Not applicable.

\section{Competing interests}

SMe: None. KB: None. RD: Lecture fees from Roche and travel grants from Biogen. BF: None. SMa: None. MAS: None. KR: None. DD: None. KN: None. DS: 
None. HU: Shareholder of the Veobrain: HP: None. KD: Steering Committee Neurosciences, Janssen. LTVE: Advisory boards, lectures, or travel grants within the last three years: Roche, Eli Lilly, Janssen-Cilag, Novartis, Shire, UCB, GSK, Servier, Janssen and Cyberonics. DE: None.

\section{Author details}

1 Section for Experimental Neuropsychiatry, Department of Psychiatry and Psychotherapy, Medical Center-University of Freiburg, Faculty of Medicine, University of Freiburg, Freiburg, Germany. ${ }^{2}$ Department of Psychiatry and Psychotherapy, Medical Center-University of Freiburg, Faculty of Medicine, University of Freiburg, Freiburg, Germany. ${ }^{3}$ Department for Psychiatry and Psychotherapy II, Ulm University, Bezirkskrankenhaus Günzburg, Günzburg, Germany. ${ }^{4}$ Department of Neurology, Medical Center-University of Freiburg, Faculty of Medicine, University of Freiburg, Freiburg, Germany. ${ }^{5}$ Department of Psychosomatic Medicine and Psychotherapy, Medical Center-University of Freiburg, Faculty of Medicine, University of Freiburg, Freiburg, Germany. ${ }^{6}$ Department of Neuroradiology, Medical Center-University of Freiburg, Faculty of Medicine, University of Freiburg, Freiburg, Germany. ${ }^{7}$ Department of Neurology and Experimental Neurology, Charité-Universitätsmedizin Berlin, Berlin, Germany. ${ }^{8}$ German Center for Neurodegenerative Diseases (DZNE), Berlin, Germany. ${ }^{9}$ Center for Basics in Neuromodulation, Faculty of Medicine, University of Freiburg, Freiburg, Germany.

\section{Received: 1 August 2020 Accepted: 6 October 2020}

\section{Published online: 11 November 2020}

\section{References}

1. Deisenhammer F, Bartos A, Egg R, Gilhus NE, Giovannoni G, Rauer S, Sellebjerg F. Guidelines on routine cerebrospinal fluid analysis. Report from an EFNS task force. Eur J Neurol. 2006;13(9):913-22. https://doi.org/10.111 1/j.1468-1331.2006.01493.x.

2. PollakTA, Lennox BR, Muller $S$, Benros ME, Pruss H, Tebartz van Elst $L$, Klein H, Steiner J, Frodl T, Bogerts B, Tian L, Groc L, Hasan A, Baune BT, Endres D, Haroon E, Yolken R, Benedetti F, Halaris A, Meyer JH, Stassen H, Leboyer M, Fuchs D, Otto M, Brown DA, Vincent A, Najjar S, Bechter K. Autoimmune psychosis: an international consensus on an approach to the diagnosis and management of psychosis of suspected autoimmune origin. Lancet Psychiatry. 2020;7(1):93-108. https://doi.org/10.1016/s2215 -0366(19)30290-1.

3. Banks WA, Erickson MA. The blood-brain barrier and immune function and dysfunction. Neurobiol Dis. 2010;37(1):26-322. https://doi. org/10.1016/j.nbd.2009.07.031.

4. Carvey PM, Hendey B, Monahan AJ. The blood-brain barrier in neurodegenerative disease: a rhetorical perspective. J Neurochem. 2009;111(2):291-314. https://doi.org/10.1111/j.1471-4159.2009.06319.x.

5. Serlin Y, Shelef I, Knyazer B, Friedman A. Anatomy and physiology of the blood-brain barrier. Semin Cell Dev Biol. 2015;38:2-6. https://doi. org/10.1016/j.semcdb.2015.01.002.

6. Spieler D, Namendorf C, Namendorf T, von Cube M, Uhr M. Donepezil, a cholinesterase inhibitor used in Alzheimer's disease therapy, is actively exported out of the brain by abcb1ab p-glycoproteins in mice. J Psychiatr Res. 2020;124:29-33. https://doi.org/10.1016/j.jpsychires.2020.01.012 (Epub 2020 Jan 27).

7. Pollak TA, Drndarski S, Stone JM, David AS, McGuire P, Abbott NJ. The blood-brain barrier in psychosis. Lancet Psychiatry. 2018;5(1):79-92. https ://doi.org/10.1016/s2215-0366(17)30293-6.

8. Brochner CB, Holst CB, Mollgard K. Outer brain barriers in rat and human development. Front Neurosci. 2015;9:75. https://doi.org/10.3389/fnins .2015 .00075 .

9. Engelhardt B, Sorokin L. The blood-brain and the blood-cerebrospinal fluid barriers: function and dysfunction. Semin Immunopathol. 2009;31(4):497-511. https://doi.org/10.1007/s00281-009-0177-0.

10. Erickson MA, Banks WA. Neuroimmune axes of the blood-brain barriers and blood-brain interfaces: bases for physiological regulation, disease states, and pharmacological interventions. Pharmacol Rev. 2018;70(2):278-314. https://doi.org/10.1124/pr.117.014647.

11. Erickson MA, Nicolazzo JA, Banks WA. Commentary on the 2018 Named Series on blood-brain interfaces: roles of neuroimmunomodulation in health and disease. Brain Behav Immun. 2018;74:3-6. https://doi. org/10.1016/j.bbi.2018.08.016.

12. Tumani $H$, Huss $A$, Bachhuber F. The cerebrospinal fluid and barriers - anatomic and physiologic considerations. Handb Clin Neurol. 2017;146:21322. https://doi.org/10.1016/b978-0-12-804279-3.00002-2.

13. Wildemann B, Oschmann P, Reiber H. Laboratory diagnosis in neurology 1st. edition ed. Stuttgart: Thieme; 2010.

14. Reiber H. Flow rate of cerebrospinal fluid (CSF)-a concept common to normal blood-CSF barrier function and to dysfunction in neurological diseases. J Neurol Sci. 1994;122(2):189-203. https://doi.org/10.1016/0022510x(94)90298-4.

15. Reiber H, Uhr M. Physiologie des Liquors. In: Berlit P, editor. Klinische Neurologie. Berlin: Springer Berlin Heidelberg; 2018. p. 1-19.

16. Ahn SM, Byun K, Cho K, Kim JY, Yoo JS, Kim D, Paek SH, Kim SU, Simpson RJ, Lee B. Human microglial cells synthesize albumin in brain. PLoS ONE. 2008;3(7):e2829. https://doi.org/10.1371/journal.pone.0002829.

17. Park JH, Park JA, Ahn JH, Kim YH, Kang IJ, Won MH, Lee CH. Transient cerebral ischemia induces albumin expression in microglia only in the CA1 region of the gerbil hippocampus. Mol Med Rep. 2017;216(1):661-5. https://doi.org/10.3892/mmr.2017.6671 (Epub 2017 May 31).

18. Castellazzi M, Morotti A, Tamborino C, Alessi F, Pilotto S, Baldi E, Caniatti LM, Trentini A, Casetta I, Granieri E, Pugliatti M, Fainardi E, Bellini T. Increased age and male sex are independently associated with higher frequency of blood-cerebrospinal fluid barrier dysfunction using the albumin quotient. Fluids Barriers CNS. 2020. https://doi.org/10.1186/ s12987-020-0173-2.

19. Parrado-Fernandez C, Blennow K, Hansson M, Leoni V, Cedazo-Minguez A, Bjorkhem I. Evidence for sex difference in the CSF/plasma albumin ratio in 20000 patients and 335 healthy volunteers. J Cell Mol Med. 2018;22(10):5151-4. https://doi.org/10.1111/jcmm.13767.

20. Endres D, Meixensberger S, Dersch R, Feige B, Stich O, Venhoff N, Matysik M, Michel M, Runge K, Nickel K, Urbach H, Domschke K, Prüss H, Tebartz van Elst L. Cerebrospinal fluid, antineuronal autoantibody, EEG, and MRI findings from 992 patients with schizophreniform and affective psychosis. Transl Psychiatry. 2020;10(1):279. https://doi.org/10.1038/s4139 8-020-00967-3.

21. Engelborghs S, Niemantsverdriet E, Struyfs $H$, Blennow K, Brouns R, Comabella M, Dujmovic I, van der Flier W, Frolich L, Galimberti D, Gnanapavan S, Hemmer B, Hoff E, Hort J, lacobaeus E, Ingelsson M, Jan de Jong F, Jonsson M, Khalil M, Kuhle J, Lleo A, de Mendonca A, Molinuevo JL, Nagels G, Paquet C, Parnetti L, Roks G, Rosa-Neto P, Scheltens P, Skarsgard C, Stomrud E, Tumani H, Visser PJ, Wallin A, Winblad B, Zetterberg H, Duits F, Teunissen CE. Consensus guidelines for lumbar puncture in patients with neurological diseases. Alzheimers Dement. 2017;8:111-26. https:// doi.org/10.1016/j.dadm.2017.04.007.

22. Stich $\mathrm{O}$, Rauer $\mathrm{S}$, Kaiser R Liquordiagnostik. In Neurologie compact. Für Klinik und Praxis. Hufschmidt A, Lücking CH, Rauer S. 2013. 6th edition, Thieme, Stuttgart. DOI: 10.1055/b-0034-66239

23. R Development Core Team (2008). R: A language and environment for statistical computing. R Foundation for Statistical Computing, Vienna. ISBN 3-900051-07-0, https://www.r-project.org.

24. Weaving G, Batstone GF, Jones RG. Age and sex variation in serum albumin concentration: an observational study. Ann Clin Biochem. 2016;53(Pt 1):106-11. https://doi.org/10.1177/0004563215593561.

25. McCudden CR, Brooks J, Figurado P, Bourque PR. Cerebrospinal fluid total protein reference intervals derived from 20 years of patient data. Clin Chem. 2017;63(12):1856-65. https://doi.org/10.1373/clinc hem.2017.278267 (Epub 2017 Oct 11).

26. Castellazzi M, Pizzicotti S, Lombardo I, Alfiero S, Morotti A, Pellegatti P, Negri G, Natali L, Ferri C, Fainardi E, Bellini T, Pugliatti M. Sexual dimorphism in the cerebrospinal fluid total protein content. Clin Chem Lab Med. 2020. https://doi.org/10.1515/cclm-2020-0419 (Online ahead of print).

27. Reiber H. Proteins in cerebrospinal fluid and blood: barriers, CSF flow rate and source-related dynamics. Restor Neurol Neurosci. 2003;21(3-4):79-96.

28. Seyfert S, Faulstich A. Is the blood-CSF barrier altered in disease? Acta Neurol Scand. 2003;108(4):252-6. https://doi.org/10.103 4/j.1600-0404.2003.00119.x.

29. Bechter K. The peripheral cerebrospinal fluid outflow pathway Physiology and pathophysiology of CSF recirculation: A review and 
hypothesis. Neurol Psychiatry Brain Res. 2011. https://doi.org/10.1016/j. npbr.2011.06.003ss.

30. Bechter K, Schmitz B. Cerebrospinal fluid outflow along lumbar nerves and possible relevance for pain research: case report and review. Croatian Med J. 2014;55(4):399-404. https://doi.org/10.3325/cmj.2014.55.399.

31. Bechter $K$, Hof PR, Benveniste $H$. On the flow dynamics of cerebrospinal fluid. Neurol Psychiatry Brain Res. 2015;21 (2):96-103. https://doi. org/10.1016/j.npbr.2015.01.001.

32. Benveniste $H$, Hof PR, Nedergaard M, Bechter K. Modern cerebrospinal fluid flow research and Heinrich Quincke's seminal 1872 article on the distribution of cinnabar in freely moving animals. J Comp Neurol. 2015;523(12):1748-55. https://doi.org/10.1002/cne.23758.

33. Maggioli E, McArthur S, Mauro C, Kieswich J, Kusters DH, Reutelingsperger CP, Yaqoob M, Solito E. Estrogen protects the blood-brain barrier from inflammation-induced disruption and increased lymphocyte trafficking. Brain Behav Immun. 2016;51:212-22. https://doi.org/10.1016/j. bbi.2015.08.020.

34. Brettschneider J, Claus A, Kassubek J, Tumani H. Isolated blood-cerebrospinal fluid barrier dysfunction: prevalence and associated diseases. J Neurol. 2005;252(9):1067-73. https://doi.org/10.1007/s00415-005-0817-9.

35. Gudmundsson P, Skoog I, Waern M, Blennow K, Zetterberg H, Rosengren $\mathrm{L}$, Gustafson D. Is there a CSF biomarker profile related to depression in elderly women? Psychiatry Res. 2010;176(2-3):174-8. https://doi. org/10.1016/j.psychres.2008.11.012 (Epub 2010 Feb 4).

36. Zetterberg H, Jakobsson J, Redsäter M, Andreasson U, Pålsson E, Ekman CJ, Sellgren C, Johansson AG, Blennow K, Landén M. Blood-cerebrospinal fluid barrier dysfunction in patients with bipolar disorder in relation to antipsychotic treatment. Psychiatry Res. 2014;217(3):143-6. https://doi. org/10.1016/j.psychres.2014.03.045 (Epub 2014 Apr 5).

37. Skillbäck T, Delsing L, Synnergren J, Mattsson N, Janelidze S, Nägga K, Kilander L, Hicks R, Wimo A, Winblad B, Hansson O, Blennow K, Eriksdotter $\mathrm{M}$, Zetterberg $\mathrm{H}$. CSF/serum albumin ratio in dementias: a cross-sectional study on 1861 patients. Neurobiol Aging. 2017;59:1-9. https://doi. org/10.1016/j.neurobiolaging.2017.06.028 (Epub 2017 Jul 11).

38. Orlovska-Waast S, Köhler-Forsberg O, Brix SW, Nordentoft M, Kondziella D, Krogh J, Benros ME. Cerebrospinal fluid markers of inflammation and infections in schizophrenia and affective disorders: a systematic review and meta-analysis. Mol Psychiatry. 2019;24(6):869-87. https://doi. org/10.1038/s41380-018-0220-4
39. Musaeus CS, Gleerup HS, Høgh P, Waldemar G, Hasselbalch SG, Simonsen AH. Cerebrospinal fluid/plasma albumin ratio as a biomarker for blood-brain barrier impairment across neurodegenerative dementias. J Alzheimers Dis. 2020;75(2):429-36. https://doi.org/10.3233/JAD-200168.

40. Maxeiner HG, Rojewski MT, Tumani H, Herzog S, Fuchs D, Schmitt A, Schmitt M, Bechter K. Immunological and histochemical analyses of cerebrospinal fluid and peripheral blood from patients with neurological and psychiatric disorders. Acta Neuropsychiatr. 2009;21(Suppl 2):51-7. https://doi.org/10.1017/s0924270800032737.

41. Bechter K, Reiber H, Herzog S, Fuchs D, Tumani H, Maxeiner HG. Cerebrospinal fluid analysis in affective and schizophrenic spectrum disorders: identification of subgroups with immune responses and blood-CSF barrier dysfunction. J Psychiatr Res. 2010;44(5):321-30. https://doi. org/10.1016/j.jpsychires.2009.08.008.

42. Bechter K. CSF diagnostics in psychiatry-present status-future projects. Neurol Psychiatry Brain Res. 2016. https://doi.org/10.1016/j. npbr.2016.01.008

43. Endres D, Dersch R, Hottenrott T, Perlov E, Maier S, van Calker D, Hochstuhl B, Venhoff N, Stich O, van Elst LT. Alterations in cerebrospinal fluid in patients with bipolar syndromes. Front Psychiatry. 2016;7:194. https://doi. org/10.3389/fpsyt.2016.00194.

44. Endres D, Perlov E, Baumgartner A, Hottenrott T, Dersch R, Stich O, Tebartz van Elst L. Immunological findings in psychotic syndromes: a tertiary care hospital's CSF sample of 180 patients. Front Hum Neurosci. 2015;9:476. https://doi.org/10.3389/fnhum.2015.00476.

45. Endres D, Perlov E, Dersch R, Baumgartner A, Hottenrott T, Berger B, Stich $\mathrm{O}$, Tebartz van Elst L. Evidence of cerebrospinal fluid abnormalities in patients with depressive syndromes. J Affect Disord. 2016;198:178-84. https://doi.org/10.1016/j.jad.2016.03.030.

46. Bechter K, Deisenhammer F. Psychiatric syndromes other than dementia. Handb Clin Neurol. 2017;146:285-96. https://doi.org/10.1016/b978-0-12804279-3.00017-4.

\section{Publisher's Note}

Springer Nature remains neutral with regard to jurisdictional claims in published maps and institutional affiliations.
Ready to submit your research? Choose BMC and benefit from:

- fast, convenient online submission

- thorough peer review by experienced researchers in your field

- rapid publication on acceptance

- support for research data, including large and complex data types

- gold Open Access which fosters wider collaboration and increased citations

- maximum visibility for your research: over $100 \mathrm{M}$ website views per year

At BMC, research is always in progress.

Learn more biomedcentral.com/submissions 